\title{
How many fish make a mouse?
}

\author{
How do we measure the impact of scientific research? A new study discusses the current publication culture, \\ diverse animal models that are commonly used in cardiovascular studies, the comparison between basic and \\ clinical research paths, and the role of authors and reviewers in bringing these two paths together.
}

\section{Eldad Tzahor and Karina Yaniv}

3 ublishing research is an important requirement for an academic career and it is often used as a measure of professional success. Early in our academic training, we hear 'the mantra' that the impact factor of the outlet where studies are published can make or break a career. Not only does the pressure to publish regularly and in highly visible journals start at the onset of a research career, in fact, it never stops. How well and often we publish drastically affects our ability to get the funding needed to support the research we plan to do.

We often ask ourselves, what is the magic ingredient that adds that little something to a study and qualifies it for publication in a top-tier journal? One would expect that it is the leap in knowledge that the new research provides, which would then inspire and steer the field. However, less clarity exists about how much data is needed to support the main conclusions during the process of manuscript preparation. This lack of clarity becomes particularly evident for those of us focused on basic science and working with model organisms that are evolutionally distant from humans. How many times have we received reviewer comments challenging the main conclusions of our research by questioning the relevance of zebrafish studies to humans, or asking whether similar mechanisms exist in mammals? Even extensive research carried out in animals that are evolutionary closer to humans, such as mice and rats, is not always enough, especially for studies with potential clinical implications. As one reviewer bluntly commented, "this is another study curing mice, which is often not replicated in large animals or clinical studies".

The price we are often asked to pay is reflected in the amount of data required for publication, which seems to be inversely proportional to the perceived relevance of the model organism. To make it simple, the value of the results boils down to how close the model organism is to humans. With this artificial relevance ladder, we find ourselves constantly trying to figure out how many fish would be enough to make up for a mouse', or 'how many mice would equal one pig', a large-animal model preferred in the preclinical arena for cardiac studies.

Translational therapeutic approaches depend on basic research, which is performed primarily in animal models. Although neither part is ideal alone or able to substitute for human studies, it is clear that several groundbreaking discoveries were made in organisms that are evolutionally very distant from humans, and were only years later recapitulated in higher organisms. Let us look, for instance, at the field of heart regeneration. Although the textbooks argued that the heart is an organ that does not regenerate following injury, the seminal findings by Poss and Keating ${ }^{1}$, published 20 years ago, demonstrated that zebrafish hearts do possess a significant regenerative capability. The relevance to mammals was not demonstrated back then, but only a decade later, when Porello, Olson and Sadek showed that the same principle holds true for neonatal mice ${ }^{2}$. Subsequently, these findings were replicated in newborn pigs $^{3,4}$ and suggested to occur also in human newborns ${ }^{5,6}$. This is a clear example of a bottom-up knowledge foundation that pushed the field forward, and still drives researchers and biotechnology companies to seek mechanisms underlying the innate regenerative response in vertebrates and ultimately find ways to boost this response in human patients.

The field of lymphatic research represents another example of the crucial contribution of basic science. Lymphatic vessel malfunction is associated with pathogenesis of many diseases, including lymphedema, fibrosis and inflammation. In malignancies, the formation of tumor-associated lymphatic vessels correlates with metastatic dissemination, increased severity and poor patient prognosis. Conversely, the presence of functional lymphatics is regarded as beneficial for anti-tumor immunity, including cancer immunotherapy ${ }^{8,9}$. One of the major bottlenecks in the field of lymphatic research was (and still is) the understanding of where lymphatic vessels come from. This question has been debated for more than a century and has involved an incredible number of animal models, including zebrafish, mouse, chick, trout and even turtle ${ }^{10}$. Collectively, these animal models shaped the current view of lymphatic vessel origins, highlighting their unexpected plasticity with consequent physiological and pathological effects in humans. Finally, the recent discovery of the lymphatic system of the central nervous system - first in mice $^{11,12}$, and soon after in humans ${ }^{13}-$ opened up a new field of research focused on the function of lymphatic vessels in the human brain and made us rethink our approach to neurodegenerative diseases ${ }^{14,15}$.

The value of basic research is widely accepted but not always sufficiently appreciated. This has been particularly highlighted in the past couple of years, owing to the rapid development of the COVID-19 vaccines, designed to curb and hopefully stop the pandemic and its devastating effects. Getting several designs of this vaccine in such a short period of time was possible only because of decades of basic research studies involving animal models that, at the time, lacked clinical relevance, but, importantly, laid down the necessary foundation for vaccine development. Despite the crucial importance of basic science, it appears that fundamental research is held to higher standards in receiving funding, and subject to greater scrutiny during the publication process, than translational and clinical studies. Publishing basic research often includes revisions that are long and costly because it is requested to show relevance to mammals (for zebrafish studies) or to large animals or non-human primates (for mouse studies).

The use of diverse animal models has been crucial to drive the field of cardiovascular research forward by establishing novel concepts and paradigms, and suggesting molecular targets for therapeutic approaches. Yet, it is important to keep in mind that the path from basic research to the clinic is not linear - it involves asking difficult and provocative questions and formulating hypotheses that require many years of research to be 
answered properly. This process often begins with fundamental discoveries based on research using what are considered to be low, primitive or simple animal models. Sometimes, it takes decades until further experimentation, modeling and testing in large animal models yields a therapeutic agent and proves the initial finding's 'worth to humans. Therefore, it is not reasonable to expect that this entire trajectory is included in a single publication. Instead, we should focus on the impact of the findings themselves and on the new avenues of research that they might open.

There is no doubt that basic research could be optimized to yield better clinical translation. However, should this be what guides us as basic scientists? Or, equally importantly, as reviewers? Because let us not forget that authors and reviewers are one and the same - the foundation of the 'peer' review process.

How do we measure the relevance of a scientific study or its impact? Currently, there seems to be a clear drift towards clinical and translational science, which is reflected in publication opportunities and the impact of the scientific journals where such research is published. Instead, should the real impact of a study not be defined as 'research that inspires more research'? Let us now make a full circle - if the initial zebrafish heart regeneration study was ignored or published in a poorly visible journal because it lacked confirmation in mammals, we may not have reached the stage two decades later in which drugs are being tested to promote cardiac repair in clinical trials.

To push the boundaries of cardiovascular science harder and stronger, it is important that basic and clinical research work hand in hand. Instead, these two communities seem to communicate poorly, and this is particularly obvious in scientific meetings and publishing outlets that struggle to bring these two communities together. Combined meeting agendas, publishing platforms and funding opportunities for researchers in both fields would be instrumental to improve the current situation in cardiovascular (or any other type of) research. In addition, let us remind ourselves that the real power for changing the publication culture lies in our hands. Both as authors and as reviewers, we have the opportunity and responsibility to evaluate each study based on the extent to which it expands our knowledge, and not whether it possesses imminent translational potential and demonstrable relevance to higher organisms that include humans.

At the end, it is not the equation of 'how many fish make a mouse?' that leads to effective translational discoveries, but rather the collection of research breakthroughs and acquired knowledge that would be ultimately applied to improve human health and quality of life.

\section{Eldad Tzahor (D) $1 \square$ and Karina Yaniv (D) $2 \square$}

${ }^{1}$ Department of Molecular Cell Biology, Weizmann Institute of Science, Rehovot, Israel. ${ }^{2}$ Department of Biological Regulation, Weizmann Institute of Science, Rehovot, Israel.

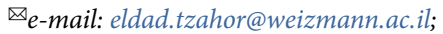

karina.yaniv@weizmann.ac.il

Published online: 12 January 2022

https://doi.org/10.1038/s44161-021-00010-8

References

1. Poss, K. D., Wilson, L. G. \& Keating, M. T. Science 298, 2188-2190 (2002).

2. Porrello, E. R. et al. Science 331, 1078-1080 (2011).

3. Zhu, W. et al. Circulation 138, 2809-2816 (2018).

4. Ye, L. et al. Circulation 138, 2798-2808 (2018)

5. Polizzotti, B. D. et al. Sci. Transl. Med. 7, 281 ra45 (2015).

6. Sadek, H. \& Olson, E. N. Cell Stem Cell 26, 7-16 (2020).

7. Tzahor, E. \& Poss, K. D. Science 356, 1035-1039 (2017).

8. Oliver, G. et al. Cell 182, 270-296 (2020).

9. Alitalo, K. Nat. Med. 17, 1371-1380 (2011).

10. Semo, J., Nicenboim, J. \& Yaniv, K. Development 143, 924-35 (2016).

11. Louveau, A. et al. Nature 523, 337-41 (2015).

12. Aspelund, A. et al. J. Exp. Med. 212, 991-999 (2015).

13. Absinta, M. et al. eLife 6, e29738 (2017).

14. Louveau, A., Mesquita, S. D. \& Kipnis, J. Neuron 91, 957-973 (2016).

15. Da Mesquita, S., Fu, Z. \& Kipnis, J. Neuron 100, 375-388 (2018).

Acknowledgements

The Yaniv Lab is supported in part by European Research Council (ERC) (CoG no. 818858). K.Y. is the incumbent of the Enid Barden and Aaron J. Jade Professorial Chair. The Tzahor Lab is supported by the ERC (AdG no. 788194), EU Horizon 2020 research and innovation program REANIMA and the Israel Science Foundation.

Competing interests

The authors declare no competing interests 